Investigaciones Fenomenológicas, vol. Monográfico 5, 2015, 143-157.

e-ISSN: $1885-1088$

\title{
MODIFICACIÓN DE NEUTRALIDAD Y CRISIS DE LAS CIENCIAS \\ EUROPEAS: SOBRE LA POSIBILIDAD DE UNA VIDA DE LA RAZÓN
}

\author{
Modification Of NeUtrality AND CRISIS Of EUROPEAN \\ SCIENCES: ON THE POSSIBILITY Of A Life of ReAson
}

\author{
Joan González Guardiola \\ Universidad de Barcelona \\ Barcelona, España \\ phaenom11@hotmail.com
}

Resumen: El presente artículo pretende investigar el alcance, la función y la articulación que juega la modificación de neutralidad en "Ideas I". A través del análisis de su estructura, se llegará a la conclusión de que, en cierto sentido, la modificación de neutralidad puede suponer una temprana vía de acceso a las temáticas que años más tarde serán desarrolladas en la "Crisis de las ciencias europeas", interpretación ya sugerida por Brainard. Se sugiere, asimismo, un aumento de la presencia de los modos de la conciencia neutralizada en el quehacer matemático propio de algunas actitudes "modelizadoras" de algunas ciencias sociales.

Palabras clave: Modificación de neutralidad, reducción fenomenológico-psicológica, Crisis de las ciencias europeas, posicionalidad.

\begin{abstract}
This article aims to investigate the scope, function and articulation of neutrality modification in "Ideen I". Through the analysis of its structure, it will come to the conclusion that, in a certain sense, the neutrality modification can be an early path to the themes that years later will be developed in the "Crisis of European Sciences", as Brainard's interpretation suggest. It also comes up with an increase in the presence of modes of neutralized consciousness in own mathematical work of some "modeler" attitudes of some social sciences.
\end{abstract}

Key Words: Neutrality Modification, Phenomenological-Psychological Reduction, Crisis of European Sciences, Positionality.

En el año 1911, dos años antes de la publicación de "Ideas I", escribe Husserl lo siguiente:

Todo vivir es tomar posición (Stellungnehmen), todo tomar posición se encuentra bajo un deber (Sollen), bajo cierta jurisdicción respecto de lo que es válido o no es válido, según presuntas normas de validez absoluta. Mientras no se discutían estas normas, ni las amenazaba ni desdeñaba ningún escepticismo, no existía más que una cuestión vi- 
tal: el mejor modo de satisfacer en la práctica esas normas. Pero, ¿qué hacer ahora, cuando toda norma es discutida o empíricamente falseada y privada de su validez ideal? Los naturalistas y los historicistas luchan por la cosmovisión y sin embargo los dos acometen desde distintos lados la tarea de transmutar las ideas en hechos y de transformar toda la realidad, toda vida, en una incomprensible amalgama de "hechos" sin ideas. Ambos comparten la superstición de los hechos.

No cabe duda de que no podemos esperar. Es preciso tomar posición, tenemos que procurar mitigar las faltas de armonía en nuestra posición ante la realidad - la realidad de la vida (Lebenswirklichkeit), que tiene para nosotros significado, y en la que hemos nosotros de tener significado ${ }^{-1}$.

Lo que la lectura de este texto nos mueve a preguntar es: si todo vivir está caracterizado por una "toma de posición", ¿cómo cabe evaluar la "modificación de neutralidad", entendida en el programa de "Ideas I" como "sumamente importante" cia en general"33 ? En el texto de "Ideas I" Husserl ha presentado la modificación de neutralidad como una modificación de toda la estructura de la correlación noético-noemática en la cual "el carácter de posición (Setzungcharakter) ha perdido toda su fuerza" ${ }^{4}$. Husserl llega a afirmar que las nóesis neutralizadas no están ni siquiera sujetas a una "legitimación por la razón" (Rechtsprechung der Vernunft), y que a muy duras penas puede sostenerse que los nóemas sometidos a la modificación de neutralidad consisten en "posiciones": la intencionalidad sometida a la modificación de neutralidad no "pone" nada, no es conciencia posicional, es presentada como "mero pensar" ${ }^{5}$.

¿No anticipa ya en algún sentido la modificación de neutralidad, según la conocida interpretación de Brainard, el tema de la crisis de las ciencias europeas $?^{6}$. Y sobre todo: ¿cómo puede consistir a su vez en una posición la suspensión de todas las posiciones? ¿Cómo puede haber una "vida de la razón" basada en suspender todas las posiciones y que se mantenga, a pesar de todo, dentro del ámbito de la vida?

La legitimidad de estas preguntas no puede eliminarse fácilmente a través de matizaciones conceptuales. Aunque en "Ideas I" el vocabulario de la posicionalidad es bastante variable y diverso, y sepamos que Husserl nunca acabó de

\footnotetext{
${ }^{1}$ Vid. Klostermann, 1965, págs. 65-66. Respecto a "Stellungnehmen" Elsa Tabernig (1992) y Francesc Pereña (1999) traducen por "tomar posición", que preferimos sobre la "actitud" de Miguel García-Baró (2009).

${ }^{2}$ Hua III, § [109], pág. 222.

${ }^{3}$ Hua III, § [114], pág. 232: "grundwesentliche Eigentümlichkeit alles Bewusstsein überhaupt".

${ }^{4}$ Hua III, § [109], pág. 223.

${ }^{5}$ Hua III, § [110], pág. 223: "Hingegen das blosse Sich-denken "setzt" nichts, es ist kein positionales Bewusstsein"

${ }^{6}$ Brainard, 2002, pág. 31.
} 
encontrarse cómodo en él ${ }^{7}$, no podemos ventilar la pregunta simplemente afirmando que la expresión "Stellungnehmen" no es la habitual en los análisis husserlianos técnicos sobre la modificación de neutralidad ${ }^{8}$. De hecho, el vocabulario de la neutralidad, que nunca acabó de gustar a Husserl (dejó de utilizarlo después de 1925, fecha en la que parece ser que desplazó esta temática bajo el tratamiento de la "reducción fenomenológico-psicológica") ${ }^{9}$, se presenta en una diversidad de ámbitos temáticos bastante amplia. Encontramos usos del vocabulario de la "neutralización" en:

(a) La tendecia "no-ponente" de la percepción

(b) El objeto en conciencia de imagen neutralizado

(c) La pura (mera) fantasía

(d) La no-posicionalidad de la existencia individual en la ideación

(e) La reducción fenomenológico-psicológica

(f) La reducción trascendental

Los tres primeros son modos de ejecución (Vollzugsmoden) naturales de neutralización, y por tanto no sería correcto considerarlos en sentido estricto como "modificaciones" de neutralidad. La neutralización forma parte en ellos de una dirección de la mirada ya directa y cuasi-inmediata, de una aprehensión predestinada a la formación de juicios sobre objetos tomados en conciencia de imagen, sobre objetos en la fantasía, o sobre la percepción en tanto que motivada. Por ejemplo, en los análisis genéticos sobre la percepción que Husserl lleva a cabo en su última obra publicada, Erfahrung und Urteil, se presenta la percepción como siempre ya imbricada en un interés (Interesse) y como siempre "dirigida" (Zuwendung) hacia este ${ }^{10}$. Esta percepción, ya siempre orientada por un interés y por una aspiración (Streben), y siempre afectivamente acompañada, tiene un objeto (Gegenstand), que constituye el tema (Thema) de la percepción ${ }^{11}$. Cuando un objeto es temático, permanece siempre vuelto en cierto sentido hacia el yo que lo percibe y su consubstancial corriente de intereses y aspiraciones. Un objeto no temático es un objeto de la percepción que está

\footnotetext{
7 Hua XXIII, Apéndice XLVIII, pág. 459: "Yo utilizaba "actual - inactual", también "ponente-no ponente", o "posicional-neutral", iuna terminología bastante mala! (lauter schlechte)" (trad. nuestra).

${ }^{8}$ La expresión sí aparece en "Ideas I" (Hua III, § [37], pág. 77; § [38], pág. 78; § [80], pág. 179; § [31], pág. 63), y aunque menos habitual no parece guardar un significado distinto a las utilizadas de modo más técnico.

${ }^{9}$ Vid. Liangkang $\mathrm{Ni}, 1999$, pág. 194.

${ }^{10}$ Erfahrung und Urteil, a partir de ahora EU, § [20].

11 Ibid, pág. 91: "Mit diesem Streben Hand in Hand ein Gefühl geht".
} 
fuera de esa orientación hacia el yo y su corriente de aspiraciones. Si estoy implicado en un trabajo científico y me distrae un ruido de la calle, este ruido de la calle es un objeto de mi percepción, pero no es un objeto temático de mi acto perceptivo. Mientras desplazo mi atención a los ruidos de la calle, el objeto de mi trabajo científico no pierde su carácter temático. La percepción de los ruidos de la calle que han acabado distrayéndome no forma parte de la corriente de intereses y aspiraciones del yo percipiente. Cuando vuelvo la atención de nuevo hacia mi trabajo científico, los ruidos de la calle pueden seguir ahí, pero no forman parte de mi percepción temática ${ }^{12}$. Esta percepción "no temática" es percepción "no ponente", en el sentido que sus objetos no forman parte de la "tendencia hacia el yo" de la percepción temática. Este sentido de "no posicionalidad" se mezcla con el sentido que Husserl ya tenía en "Ideas I" respecto a los modos de conciencia actuales e inactuales, ponentes-no ponentes, y con la temática de los modos de ser del horizonte en el despliegue posterior de la fenomenología trascendental ${ }^{13}$ : estos sentidos no remiten a una modificación de neutralidad de algo dado primeramente como no-neutral, sino a modos de ser de la conciencia propios que implican de alguna manera elementos no-ponentes en los modos de la percepción.

Algo muy parecido sucede con el objeto en conciencia de imagen o el objeto de la pura fantasía. Cuando percibo un cuadro, percibo un lienzo en el cual se extienden líneas formando figuras y algunas superficies coloreadas. Este es el contenido de la percepción que Husserl llama "normal". Pero el cuadro no es un objeto normal, es percibido como un objeto-imagen (Bildobjekt), y el acceso inmediato que tenemos a él se lleva a cabo a través de la conciencia de imagen, es decir, orientada ya directamente, no a los objetos de la percepción normal, sino a los objetos que éstos, a través de la semejanza, figuran (abbilden $)^{14}$. Esta es la presentación primaria de los objetos-imagen, y la conciencia debería hacer un cambio de orientación para ver los elementos del objetoimagen como objetos de una percepción normal. Con todo, los contenidos de la percepción normal están en todo momento ya en la presentación primaria del objeto-imagen, aunque están neutralizados. Las líneas y los colores, en tanto que líneas y colores, no son ni existentes ni no existentes, son simplemente

\footnotetext{
12 Siguiendo el célebre ejemplo de $E U$, pág. 92.

13 Hua III, § [35], § [122]; Hua VI, § [37].

${ }^{14}$ Vid. el clásico ejemplo del grabado de Durero, en Hua III, § [111], pág. 252.
} 
objetos neutralizados. Aunque Husserl utilice para el ejemplo de la conciencia de imagen la expresión de "modificación" de neutralidad, insistimos en que es un uso poco afortunado, y debería simplemente hablarse de "neutralización primaria", dado que es otro ejemplo en el cual la neutralización no sucede a la presentación de algo previamente dado a la conciencia como "no neutralizado", o "posicional", sino que es un carácter propio de la conciencia de algunos tipos de objeto peculiares, cuya donación está ya basada de raíz en la copresentación de elementos neutralizados ${ }^{15}$. Lo mismo podría repetirse respecto a los objetos de la mera fantasía (blosse Phantasie), que son considerados como "representaciones ponentes neutralizadas". Si el recuerdo (Erinnerung) es considerado por Husserl como el ejemplo clásico de representación ponente (setzenden Vergegenwärtigung), la fantasía no consiste en otra cosa que en la eliminación, a estas representaciones, de su carácter "ponente". Aquí "ponente" refiere, en la terminología de Husserl, a "dóxico", en tanto que una fantasía es puesta como fantasía (es un acto tético), pero esta posición no implica creencia real y efectiva, o "certeza" del ser real de su contenido. Un acto de la fantasía es un acto tético, pero no dóxico (hay, efectivamente, posicionalidad de la fantasía como "ser-fantasía", pero no hay posicionalidad del contenido de la vivencia como existiendo realmente). La fantasía se distingue de la modificación de neutralidad porque precisamente en la modificación de neutralidad la distinción entre tético y dóxico se desdibuja absolutamente. Una vivencia de fantasía neutralizada y una vivencia de percepción neutralizada son indistinguibles una vez sus contenidos y sus estructuras de correlación noético-noemáticas han sido modificadas en tanto que neutrales. Ambas son "mero pensar", "sombras de actos $^{16}$, o, como Husserl los designará más técnicamente, "quasi-actos"17. Pero a veces tendemos a decir que las vivencias de la fantasía son "no-ponentes" de los contenidos que representan; cuando así hablamos, lo hacemos en el sentido en que no creemos que las representaciones correspondan a la realidad, no creemos en ellas como reales. Sólo en este sentido, restringido a lo dóxico, hablamos de la fantasía como de una suspensión de la posicionalidad, pero co-

\footnotetext{
${ }^{15}$ Sería una explicación absurda pensar que "primero" vemos líneas y colores y "después" pasamos a ver aquello que estas líneas y colores reproducen; en el caso de la conciencia de imagen, prácticamente tengo que hacer el recorrido opuesto: tengo que forzar una dirección de la mirada para ver la imagen como un conjunto de líneas y colores, y no como un paisaje, por ejemplo.

${ }^{16}$ Hua III, § [114], pág. 259, 261.

${ }^{17}$ Hua III, § [113], pág. 256.
} 
mo Husserl se dedica a precisar con exactitud, en sentido más amplio hay que distinguir con exactitud el modo de conciencia de la fantasía respecto al modo de conciencia propio de la modificación de neutralidad.

Estos tres modos no interesan a nuestro cuestionamiento actual. Tienen su importancia respecto a nuestra temática, en tanto que implican el reconocimiento de que nuestra conciencia toda está "cruzada" por elementos neutralizados, necesarios en la fundación de modos genéricos de conciencia (la fantasía, la conciencia de imagen, la percepción no temática). En tanto que estos modos de conciencia contienen elementos neutralizados en sus estructuras noético-noemáticas más básicas, podemos decir que la neutralización no es un aspecto extraño a la vida de conciencia; al contrario, la vida de conciencia en general se muestra ya toda ella atravesada, $\operatorname{cruzada}^{18}$, por elementos neutralizados, y la neutralización como posibilidad de la conciencia no debe ser vista como un elemento extraño a la descripción de la conciencia en general.

Algo completamente distinto sucede con lo que, en un sentido más restringido, deberíamos denominar "modificación" de neutralidad. Aunque Husserl a menudo no lleve a cabo la restricción con que operaremos aquí, nos referimos con la expresión "modificación de neutralidad" tan solo a procesos aplicables al noema "mundo"19. Es decir, no tenemos en cuenta las neutralizaciones que algunos modos de conciencia necesitan como partes imprescindibles de sus procesos de constitución (partes para las que alguna vez, en un sentido más amplio, Husserl utiliza también la expresión "modificación"). En síntesis: con la expresión "modificación de neutralidad" nos referimos a un acto de conciencia que: (a) aparece en la reflexión, (b) tiene un fuerte sentido metódico, (c) no consiste en ser parte de otros modos específicos de conciencia, sino que atraviesa la conciencia toda como modificación "universal", (d) puede aplicarse al noema "mundo" como correlato total de las vivencias de la conciencia en general. En este sentido más restringido, la modificación de neutralidad, en tanto que afecta a toda la vida de la conciencia, ha tendido a ser asimilada al futuro concepto husserliano de la reducción fenomenológico-psicológica. Toda la realidad puede ser reducida a la totalidad de la vida de conciencia entendida como psíquica, todo lo dado como realidad externa física puede ser comprendido co-

\footnotetext{
${ }^{18}$ La expresión que utiliza Husserl es "durchsetzt"; vid. Hua III, § [114], pág. 259.

19 Seguimos, en esta restricción, a Liangkang Ni, 1999, pág. 195 y ss., que distingue entre un concepto amplio (weiteren Sinn) y un concepto restringido (engeren Sinn) de la expresión "modificación".
} 
mo puros contenidos de conciencia, entendiendo aquí la "conciencia" como "lo psíquico", en tanto que opuesto a "lo físico"20.

Pero, ¿cuál es la legitimación de esta asimilación? ¿Es tan lógico y natural asimilar la modificación de neutralidad, tal y como es presentada en "Ideas I", a la temática posterior de la reducción fenomenológico-psicológica? ¿O por el contrario podemos encontrar en la presentación de la modificación de neutralidad en "Ideas I" diferencias importantes respecto a lo que después será presentado bajo el título de la reducción fenomenológico-psicológica?

La reducción fenomenológico-psicológica es presentada como una reducción en la cual la psicología fenomenológica asienta la esencia de su objeto de estudio, entendido éste como "lo psíquico"21. Nada de esta referencia a lo psíquico está referida a las descripciones de la modificación de neutralidad en "Ideas I". El ámbito de exposición de la modificación de neutralidad se halla, dentro del plan de "Ideas I", en el capítulo IV de la Sección tercera, y el capítulo trata sobre "la problemática de las estructuras noético-noemáticas". No se encuentra en "Ideas I" nada de la modificación de neutralidad en el capítulo dedicado a las reducciones, que es el capítulo IV de la Sección segunda (la famosa "meditación fenomenológica fundamental" ${ }^{22}$. Por mucho que los intérpretes nos propongan que aquello que en "Ideas I" es presentado bajo el título de "modificación de neutralidad" se desplegará en el desarrollo de la fenomenología de Husserl posteriormente como "reducción fenomenológico-psicológica", es necesario analizar con detalle el papel que ocupa la modificación de neutralidad en la estructura de "Ideas I", especialmente a la hora de poder tratar nuestras preguntas iniciales.

La modificación de neutralidad es presentada por Husserl en "Ideas I" como una novedad que nunca antes se había puesto de manifiesto ${ }^{23}$. Esta novedad hace que su exposición esté repleta de imprecisiones, y que deba ser acometida

\footnotetext{
${ }^{20}$ De esta manera, la reducción fenomenológico-psicológica se constituye como la inversa de la reducción fisicalista de las vivencias a la corporalidad extensa, propia del naturalismo.

${ }^{21}$ Esta referencia a la problemática de la delimitación temática de "lo psíquico" respecto a lo físico o corpóreo se halla en todas las exposiciones de la reducción fenomenológico-psicológica; vid. por ejemplo Hua VI, § [69], pág. 238, 252 - 254; Hua IX, § [3], pág. 283.

22 De hecho no encontramos tampoco, en la presentación de las reducciones de "Ideas I", de la distinción entre una reducción fenomenológico-psicológica y una reducción trascendental. En todo caso, si esta distinción debe presuponerse como un desarrollo posterior de lo que en "Ideas I" se presenta temáticamente estructurado de modo distinto, vale la pena llamar la atención sobre estas diferencias, a la hora de evaluar los desplazamientos en el desarrollo del "sistema" fenomenológico.

${ }^{23}$ Hua III, § [109], pág. 248.
} 
a través de una vía casi negativa (a través de "eliminaciones") ${ }^{24}$ : se accederá a la modificación de neutralidad a través de lo que ésta no es. La modificación de neutralidad no es la modificación de la asunción, no es la modificación de la negación, no es la modificación de la fantasía y no es la modificación de la conciencia de imagen (por mucho que las dos últimas, como ha sido dicho anteriormente, precisen de elementos neutralizados en sus modos originarios de presentación): Husserl irá recorriendo estos modos para ir centrando lo específico del modo de la neutralización. Pero ya antes de proceder a este recorrido podemos observar una considerable cantidad de titubeos. La modificación de neutralidad es presentada programáticamente como una modificación de los caracteres de creencia, pero inmediatamente se señala que esta modificación no pertenece de modo especial a la creencia, sino que es una modificación de conciencia general y universal. Más adelante Husserl expondrá: la modificación de neutralidad sólo puede ser acreditada (ausweisen kann) ${ }^{25}$ a través de la suspensión de la creencia (a través de las posicionalidades dóxicas), pero su alcance no se restringe a estas posicionalidades, sino a todas (es decir, a las posicionalidades "téticas"). Husserl se referirá explícitamente a la exposición en el ámbito de la esfera de las creencias como de un "rodeo" (Umweg) ${ }^{26}$. Pero, ¿porqué es necesario dar un rodeo a través de la modificación de neutralidad entendida como una modificación de los caracteres de creencia de la correlación noético-noemática, y no se presenta necesariamente la posibilidad de la modificación de neutralidad a través de cualesquiera de los modos de presentación de las vivencias de conciencia en su universalidad? ¿Por qué de este modo de presentación tan enrevesado y peculiar? ${ }^{27}$

En el $\S[117]$, el concepto de "posicionalidad" es presentado en unos términos tan amplios que es prácticamente confundido con el de "intencionalidad"28. Toda intencionalidad es posicionalidad, y toda posicionalidad lo es precisamente en tanto que le pertenece una intentio. Allí donde hay intencionalidad hay posicionalidad. Así, el concepto de posición se amplía considerablemente, y la posi-

\footnotetext{
${ }^{24}$ Ibid., "Ausscheidungen".

${ }^{25}$ Hua III, § [117], pág. 270.

${ }^{26}$ Hua III, § [268]. Es "de algún modo" (in gewisser Weise) un rodeo, pero sólo lo es "de algún modo", lo que quiere decir que "de algún modo", o "en algún sentido", no lo es, como acertadamente señala Brainard (vid. Brainard, 2002, pág. 175).

27 Una tentación inmediata que parece solucionar el problema consistiría en responder "porque Husserl no tiene todavía la reducción fenomenológico-psicológica". Pero esto no resuelve nada de la cuestión de la arquitectónica de "Ideas I", ni sobre la cuestión de la necesidad del "rodeo" expositivo.

${ }_{28}$ Hua III, § [117], pág. 269.
} 
ción de creencia (posicionalidad dóxica) pasa a ser un subgénero de las posicionalidades así ampliadas.

Al carácter de posición de un acto (de una vivencia intencional) Husserl lo llama "tesis" (de aquí deriva el adjetivo "tético", en su forma latinizada, para las posiciones en general). Veamos ejemplos de vivencias intencionales en las cuales se configuran posicionalidades diferentes:

1- Yo percibo una manzana

2- Yo imagino una manzana

3- Yo recuerdo una manzana

4- Yo percibo la imagen de una manzana

5- Yo deseo una manzana

6- Yo supongo la existencia de una manzana en mi maleta

7- Yo creo en la existencia de una manzana

, correspondientes a una percepción, una fantasía, un recuerdo, un objetoimagen, una volición, una suposición (Annehmen) y una creencia. La tesis de Husserl sobre la relación entre posiciones dóxicas y posiciones téticas es que todas las vivencias téticas pueden ser expresadas a través de vivencias dóxicas, coincidentes con ellas. Así:

1- Yo creo que percibo una manzana

2- Yo creo que imagino una manzana

3- Yo creo que recuerdo una manzana

4- Yo creo que percibo la imagen de una manzana

5- Yo creo que deseo una manzana

6- Yo creo que supongo la existencia de una manzana

7- Yo creo que creo en la existencia de una manzana

De esta manera, aunque en cierto sentido las vivencias intencionales dóxicas son un subgénero de las vivencias intencionales téticas, pensadas como posicionalidades en general, ambos conjuntos de posicionalidades son en el fondo coextensivos. Si toda tesis encierra en sí misma una tesis dóxica, las tesis dóxicas encierran en sí (véase por ejemplo la proposición 7) prototesis dóxicas (Urthese). Las prototesis dóxicas son determinadas como "certeza de la 
creencia" (Glaubensgewissheit) ${ }^{29}$. El correlato tético de las prototesis dóxicas por el lado de la posicionalidad en general es denominado por Husserl con el concepto de "posicionalidad arcóntica" (archontische Setzungscharaktere) ${ }^{30}$. En algún sentido, las posicionalidades presentan cierta estructura jerárquica. No puedo, por ley de esencia, desear una manzana que no creo que exista. Así, las vivencias emotivas, con sus consecuentes "posiciones emotivas", presuponen posiciones dóxicas. Pero la posicionalidad arcóntica es coextensiva con la prototesis dóxica.

Si el "rodeo" expositivo por las posicionalidades dóxicas no es simplemente un "rodeo", ¿por qué Husserl se empeña en encontrar una neutralización "directa", una neutralización que "salte" el rodeo a través de lo dóxico, a partir de la neutralización de la posicionalidad tética "arcóntica"? Preguntado de otra manera: ¿en qué condiciones fenomenológicamente describibles tenemos acceso a una vivencia de neutralización "directa", es decir, independiente del "rodeo" a través de lo dóxico? ${ }^{31}$.

Analicemos la tesis "Yo percibo una manzana". La neutralización de esta viviencia intencional, es decir, la modificación de esta percepción en contratesis neutralizada, no se expresa bajo la forma "Yo no percibo una manzana", que sería una posición de negación. Tampoco sería, en cierto sentido, "Yo no creo que percibo una manzana", o "No creo en la existencia de esta manzana que percibo", que implica una posición de negación de una posición dóxica. La proposición "No creo en la existencia de esta manzana que percibo" deja la percepción de la manzana, noemáticamente, en la tachadura (Durchstreichung) y noéticamente, en el tachar. La negación, según Husserl, no lo es de una afirmación (Affirmation), sino de una posición (Setzung) en el sentido ampliado de cualquier modalidad de creencia. Cuando niego un afirmar, un desear, un percibir, un dudar, e incluso un creer, niego una posición. Por tanto, la negación de un creer deriva todavía en posicionalidad. Nada de esto puede ser todavía la modificación de neutralidad ${ }^{32}$.

\footnotetext{
${ }^{29}$ Hua III, § [115], pág. 264.

${ }^{30}$ Hua III, § [117], pág. 269.

${ }^{31}$ Y que no sea propia de los modos directos de ejecución, como la conciencia de imagen o la fantasía.

32 Husserl afirma que es "muy turbador" (sehr beirrend) el hecho de que con la palabra "cierto" (gewiss) se afirme el correlato de una afirmación (Bejahung), y que cabe rechazar de plano el sentido de la expresión "cierto" como si su contrario fuera una "negación", en el sentido que supondría una posición, Hua III, § [104], pág. 241.
} 
El hecho de que la modificación de neutralidad sea ejecutable sobre cualquier vivencia en cualquier momento la desvincula de cualquier operación de actualidad-inactualidad que el vivir mismo de la conciencia efectúe en el transcurrir de su corriente. Cuando Husserl, en el § [113], quiera poner en relación la modificación de neutralidad con la distinción entre actualidad e inactualidad, o entre posicionalidad actual y potencial, constantemente operativa en el fluir de la corriente inmanente del tiempo, con el objetivo de distinguir la modificación de neutralidad de los modos potenciales de posicionalidad, nos encontraremos con la paradoja de que todos los ejemplos utilizados consistirán en los antiguos modos de ejecución directa de la conciencia que contienen partes neutralizadas y que Husserl, tan solo unos parágrafos antes, se había empeñado en distinguir analíticamente de la modificación de neutralidad: la fantasía y la conciencia de imagen. Las líneas y trazos de la percepción normal de una imagen son percibidas, en la conciencia de imagen, como "fondo" de la percepción de esta imagen. La percepción de imágenes es siempre ya percepción neutralizada, pero en cualquier momento las líneas y trazos de la imagen pueden ser atendidos como centros de la percepción, y dejar de ser fondos de la percepción de la imagen. En el momento en que esto sucede, la percepción de la imagen deja de ser actual, y pasa a ser potencial. Lo mismo sucede con los recuerdos, que pueden ser puestos en tanto que recuerdos, o representados neutralizadamente, como simples vivencias flotantes (schwebt). Pero lo que Husserl defiende en el $\S[113]$ es que la distinción entre el recuerdo posicionado y el recuerdo neutralizado flotante no es correlativa a la distinción entre recuerdos actuales y potenciales. Un recuerdo neutralizado no es un recuerdo "no atendido", no es un recuerdo existente en su ser "fondo" (Hintergrund) de otras vivencias reales presentes, sean recuerdos o percepciones. La distinción entre conciencia posicional y conciencia neutral no puede ser tratada tampoco nunca bajo el modelo de la constante movilidad de la conciencia entre contenidos actuales y fondos potenciales. No será, por tanto, el concepto de "fondo" (tampoco, diríamos, el posterior concepto más amplio de "horizonte") el que nos permita comprender de manera adecuada la relación que cabe entender entre la conciencia posicional y la conciencia neutral, dado que la constante posibilidad de volver la atención hacia los fondos de las vivencias actuales, haciendo que éstas pasen a ser potenciales, y actualizando aquellos elementos propios del fondo, es propia de ambas modalidades de conciencia: posicional y neutraliza- 
$\mathrm{da}^{33}$. Dicho de otra manera: la relación entre las vivencias posicionales y las vivencias neutralizadas no sigue el modelo de la relación general entre vivencias actuales y vivencias potenciales, o lo que es lo mismo: la relación entre conciencia posicional y conciencia neutralizada no es una relación entre conciencia actual y conciencia potencial. De aquí que cuando Husserl intenta describir con alguna concreción algún análisis de conversión de conciencia posicional a conciencia neutralizada siempre vuelva a caer del lado de lo que antes ha dicho que no era la modificación de neutralidad: fantasía, conciencia de imagen, etc., vivencias que siempre guardan una continuidad estricta respecto a las variaciones de actualidad-inactualidad de la corriente de la conciencia.

Por tanto, la modificación de neutralidad no encuentra para Husserl, en "Ideas I", una descripción concreta de su aparición, de su activación, de su razón de ser. Nos veríamos tentados a decir: de su génesis, pero no nos introduciremos en la problemática genética, en buena parte ajena a la arquitectónica de "Ideas I". En el caso de Heidegger, la experiencia de la angustia, como disposición afectiva que abre el ser en su faceta más originaria, encuentra una génesis en la teoría del útil estropeado: la ruptura del habérselas cotidiano con el útil instaura el modo teórico de aparición de la cosa (Vorhanden), un modo que interrumpe la significatividad de la cosa y que "desmundaniza" y hace extraña la presencia del Dasein en el mundo ${ }^{34}$. La experiencia de la angustia encuentra en el trabajo analítico anterior de Heidegger sobre la circunmundanidad una teoría suficiente (a pesar de sus muchos posibles cuestionamientos) sobre las condiciones de aparición de la angustia. ¿Cuáles son las condiciones de aparición de la modificación de neutralidad, entendida como modificación universal de la conciencia?

Existen dos respuestas posibles a esta cuestión. Según la primera, el vivir mismo, en su transcurrir, puede llevarnos a una neutralización de la conciencia toda bajo ciertas situaciones muy difíciles y extraordinarias de reproducción. Cuando Edipo proclama, después del testimonio del mensajero:

\footnotetext{
${ }^{33}$ En esto es esencial el anexo 55, que corrige en buena medida la fluctuación terminológica entre posiciones actuales y potenciales: "Si distingo posiciones actuales y potenciales, entonces tengo que distinguir paralelamente cuasiposiciones (neutralizadas) actuales y potenciales. A la posicionalidad corresponde la cuasiposicionalidad" (Hua III/2, Beilage 55, pág. 609). Husserl llega a utilizar, en este anexo que corrige buena parte de las imprecisiones del § [113], la expresión "posición posicional" (Positionale Setzung), para designar la posicionalidad propia de la conciencia neutralizada, dado que en ella la posicionalidad misma está vaciada, es una posición sin yo viviente posicionándose en ella.

${ }^{34}$ Heidegger, SuZ, § [40].
} 
iAy, ay! Todo se cumple con certeza. iOh, luz del día, que te vea ahora por última vez! iYo que he resultado nacido de los que no debía, teniendo relaciones con los que no podía y habiendo dado muerte a quienes no tenía que hacerlo! ${ }^{35}$,

La proclama de Edipo es continuada por una estrofa del Coro en la que se proclama:

iAh, descendencia de mortales! iCómo considero que vivís una vida igual a nada! Pues, ¿qué hombre, qué hombre logra más felicidad de la que necesita para parecerlo, para no declinar una vez que ha dado esa impresión?

En el Edipo, la experiencia de verdad desvelada en un momento puntual de la corriente de consciencia no destituye el contenido de una vivencia como falso (esto sería conciencia posicional), sino que opera una auténtica moción contra la totalidad de la corriente de conciencia en tanto que cierta. Más allá de la restauración de una situación de verdad que sustituye la anterior vida en la mentira, como recita el coro, el efecto que consigue la revelación de la verdad es el de la devaluación de toda la vida humana en la nada. Algo similar encontramos en el ciertamente trágico destino del rígido pastor protestante, Edvard Vergérus, en la película "Fanny y Alexander", de Ingmar Bergman, el cual, después de una confesión a su mujer sobre lo arraigada que su máscara de virtud y rigorismo están a su propia conciencia, confiesa, después de descubrir el odio que le tienen su hijastro y su mujer, haber alcanzado una vigilia totalmente aniquiladora, que el director sueco expresa brillantemente a través de la vinculación dramática de la lucidez con la ceguera sobrevenida por los somníferos ${ }^{36}$. Estas experiencias de lucidez radical que sumergen todo el vivir humano en las tinieblas de la irrealidad son posibles para la vida de la conciencia, aunque sin duda son escasas y extra-ordinarias, y lo son por definición ${ }^{37}$.

Según la segunda respuesta, la situación en la cual es describible la aparición de la modificación de neutralidad en la vida de la conciencia es en la reflexión. En este caso, la modificación de neutralidad tiene, toda ella, un contexto de aparición para nada neutralizado, sino muy posicional o incluso, diríamos, situacional: la polémica teórica sobre la naturaleza de lo psíquico, en el ámbito

\footnotetext{
${ }^{35}$ Edipo Rey, 1182 - 1195.

${ }^{36}$ Interpretado por el actor Jan Malmsjö.

37 La experiencia de la muerte de los cercanos, por ejemplo, nos asoma a esta "experiencia de irrealidad", en una escala menor, y referente a la realidad del otro, que motiva una reflexión sobre la consistencia de la propia realidad.
} 
de la discusión sobre la posibilidad de una psicología pura científicamente fundada. Aquí, la modificación de neutralidad no juega otro papel que el de un ejercicio, incluso, podríamos decir, el de un juego fenomenológico: ¿hasta qué punto puedo aislar lo psíquico de lo físico, invirtiendo la operación que el naturalismo hace con normalidad de aislamiento de las realidades psíquicas en el estudio de la naturaleza como totalidad física? Aquí, el juego de la neutralización se las tiene que ver con las dificultades inherentes al entrecruzamiento consustancial entre lo psíquico y lo físico que tienen modalidades enteras de la vida de la consciencia, como la conciencia de imagen, la fantasía o la percepción no temática. Pero es precisamente para evitar estas modalidades de la vida de la conciencia, en las cuales lo psíquico y lo físico se dan mezclados, que la reflexión teórica lleva a cabo la completa moción contra toda forma de posicionalidad. Sin posicionalidad, o con la posicionalidad reducida, las vivencias flotan libres de todo pespunte corporal. De ese juego (de esa futura reducción fenomenológico-psicológica) nace la "conciencia neutralizada", como contraimagen de la conciencia posicional. En este caso, como señala el propio Husserl, cabe vaciar a las expresiones "sombra", "reflejo", "imagen", cualquier matiz de negatividad, que implicaría inmediatamente posicionalidad respecto a lo negado $^{38}$. Y es aquí donde cabe interrogarse: ¿puede esta neutralización de la conciencia ser productiva? ¿Puede ella generar conocimiento a través del juego con las estructuras de actos que, neutralizados o posicionales, tienen estructura lógica y son objetivantes ${ }^{39}$ No nos referimos, con esta interrogación, a las modalidades dóxicas de la hipótesis, la suposición, etc., que son modalidades de la creencia (supongamos que $X$ es, o contemplemos la probabilidad tal de que $X$ sea ..." propias de la investigación científica. Que la mezcla entre lo neutralizado y lo posicional es productiva para la vida de la conciencia, generando modalidades enteras de la vida de la conciencia (conciencia de imagen, percepción no temática, fantasía) ya ha sido mostrado de diversas maneras, pero cabe preguntar: ¿es productiva, o puede ser productiva, la modificación de neutralidad como modalidad de la conciencia universal?

La proliferación de una nueva concepción de la modelización teórica en algunas ciencias sociales, como por ejemplo la economía, invitan a estudiar más 
a fondo una respuesta de este tipo. Estas nuevas modelizaciones son de aspectos mínimos, diminutos, de la teoría económica, y no tienen en ningún momento una tentación a la globalidad. En la constitución de estos modelos, por otro lado, no hay ningún reparo en llegar a conclusiones puramente absurdas por lo que respecta a la vida empírica: si para mostrar la posibilidad de una emergencia de la función monetaria de medio de cambio a través de una serie estocástica de intercambios es necesario modelar un mundo en el cual los agentes tienen una vida infinita y son estériles, estas condiciones (no vaciadas de significado desde la perspectiva de una antropología del capitalismo) pueden ser perfectamente aceptables ${ }^{40}$. Parece configurarse así un nuevo sentido de la palabra "modelizar", y con él un nuevo sentido de la expresión "teoría científica", para el cual la vida del pensamiento ha quedado completamente neutralizada. Pero sin duda esto sería ya objeto de investigaciones posteriores ${ }^{41}$.

\section{BiBLIOGRAFÍA}

HUSSERL, Edmund: Philosophie als strenge Wissenschaft, Klostermann Texte, 1965, Frankfurt am Main (trad. castellanas, "La filosofía, ciencia rigurosa", trad. de Miguel GarcíaBaró, Ed. Encuentro, 2009, Madrid; "La filosofía como ciencia estricta", trad. de Elsa Tabernig, ed. Almagesto, 1992, Buenos Aires; trad. catalana, "Fenomenologia", de Francesc Pereña, 1999, Edicions 62, Barcelona.

NI, Liangkang: Seinsglaube in der Phänomenologie Edmund Husserls, Phaenomenologica no 153, Kluwer, 1999, Dordrecht.

BRAINARD, Marcus: Belief and its Neutralization. Husserl's System of Phenomenology in Ideas I, SUNY Press, 2002, New York.

\footnotetext{
40 Póngase la atención en que no hablamos de una teoría del dinero (ámbito general), sino tan solo de una teoría de una de sus funciones aisladas. El nuevo sentido de la modelización teórica rehúye cualquier tendencia a situarse respecto a un problema global, y atomiza los aspectos a ser modelizados, no importando demasiado, en última instancia, la coherencia de los modelos entre sí.

${ }^{41}$ En la aplicación de la teoría de juegos a la teoría de la elección racional, propia de investigaciones fundamentales en el campo de la microeconomía, parece poder hablarse de ello.
} 